\title{
Erratum to: DRT with local contexts
}

\author{
Philippe Schlenker
}

Published online: 3 August 2011

(C) Springer Science+Business Media B.V. 2011

\section{Erratum to: Nat Lang Semantics DOI 10.1007/s11050-011-9069-7}

Under the section 3.1 Quantified statements, the last paragraph "DRT cannot derive the nuclear scope case, as we already noted. But with respect to the restrictor, it does just fine-intermediate accommodation into the restrictor yields a final meaning akin to No student who is incompetent and knows it applied for this job; this seems about right." should be replaced by "DRT cannot derive the nuclear scope case, as we already noted. But with respect to the restrictor, it does just fine-intermediate accommodation into the restrictor yields a final meaning akin to No student who is incompetent and knows it applied for this job; this seems about right. By contrast, the present approach does fine in the nuclear scope case, but it replicates Heim's predictions for the restrictor (because only local indexing is available) - and these appear to be too strong."

Under the section 3.4 Multiple indexing, the paragraph starting Heim (1983, 1992)... the last sentence "A strengthening mechanism is needed to get inference (i)" should be read as "A strengthening mechanism is needed to get inference (ii)."

The online version of the original article can be found under doi:10.1007/s11050-011-9069-7.

P. Schlenker $(\square)$

Institute Jean-Nicod, Paris, France

e-mail: philippe.schlenker@gmail.com

P. Schlenker

New York University, New York, NY, USA 